\title{
Antenna-Coupled Arrays of Voltage-Biased Superconducting Bolometers
}

\author{
Michael J. Myers ${ }^{1,4}$, Adrian T. Lee ${ }^{1,2}$, P. L. Richards ${ }^{1,3,4}$ D. Schwan ${ }^{1,4}$, \\ J. T. Skidmore ${ }^{1,4}$, A. D. Smith ${ }^{5}$, Helmuth Spieler ${ }^{2}$, Jongsoo Yoon ${ }^{1,4}$ \\ ${ }^{1}$ Department of Physics, \\ University of California, Berkeley, CA 94720 \\ ${ }^{2}$ Physics Division, Lawrence Berkeley National Laboratory \\ Berkeley, CA 94720 \\ ${ }^{3}$ Material Science Division \\ Lawrence Berkeley National Laboratory, Berkeley, CA, 94720 \\ ${ }^{4}$ Space Sciences Laboratory \\ University of California, Berkeley, CA 94720 \\ ${ }^{5}$ TRW Inc. \\ One Space Park, Redondo Beach, CA 90278
}

\begin{abstract}
We report on the development of antenna-coupled Voltage-biased Superconducting Bolometers (VSBs) which use Transition-edge Sensors (TES). Antenna coupling can greatly simplify the fabrication of large multi-frequency bolometer arrays compared to horn-coupled techniques. This simplification can make it practical to implement $1000+$ element arrays that fill the focal plane of $\mathrm{mm} / \mathrm{sub}-\mathrm{mm}$ wave telescopes. We have designed a prototype device with a double-slot dipole antenna, integrated band-defining filters, and a membrane-suspended bolometer. A test chip has been constructed and will be tested shortly.
\end{abstract}

\section{INTRODUCTION}

The $200 \mu \mathrm{m}$ to $3 \mathrm{~mm}$ wavelength range has great astronomical and cosmological significance. Science goals include characterization of the cosmic microwave background, measurement of the Sunyaev-Zel'dovich effect in galaxy clusters[1], and observations of forming galaxies[2]. Cryogenic bolometers are the most sensitive broadband detectors in this frequency range. Because single bolometer pixels are reaching the photon-noise limit for many observations, the development of large arrays will be critical for future science progress.

Voltage-biased superconducting bolometers (VSBs) which use a Transition-Edge Sensor (TES) have several advantages compared to other cryogenic bolometers. Their strong negative electrothermal feedback enhances their linearity, speed, and stability. The large noise margin of the SQUID readout enables multiplexed readout schemes, which are necessary for developing large arrays.

In this paper, we discuss the development of antenna-coupled VSBs. A planar antenna can be used to couple optical power into a bolometer. This approach offers many advantages in building a large integrated array of detectors. The antenna pattern is used to control the solid angle visible to the bolometer as with a horn antenna, but planar antennas are much simpler to fabricate in large numbers than scalar horns. The antennas, band-defining filters, and bolometers can all be integrated onto a single substrate, which will greatly simplify fabrication 
of large arrays compared to current techniques. Planar antennas are naturally polarization sensitive which is advantageous for polarization measurements, for example, of the cosmic microwave background radiation.

\section{DESIGN AND CONSTRUCTION}

Our prototype design uses a dual-slot dipole planar antenna. This antenna has a symmetric beam pattern and provides a good impedance match to microstrip transmission lines. Typically, a dielectric lens in direct contact with the antenna is used and the parameters of the lens and antenna can be tuned to provide a good match to the $\mathrm{f} / \#$ of a telescope[3]. This lens also suppresses substrate modes. A variant of the dual-slot antenna that is sensitive to two linear polarizations has been developed by Chattopadhyay and Zmuidzinas[4].

The antenna is coupled to superconducting niobium microstrip. Superconducting microstrip has very low loss for frequencies well below that corresponding to twice the gap energy of the superconductor, which is $\sim 700 \mathrm{GHz}$ for $\mathrm{Nb}[5]$. This frequency range is well suited to our design, which is intended for observations of the cosmic microwave background. The line widths for the filter were chosen to be a relatively wide $10 \mu \mathrm{m}$ to give tolerance in the fabrication process. This width gives a relatively low impedance, and we use a tapered microstrip transformer to match the antenna to the filter stage[6].
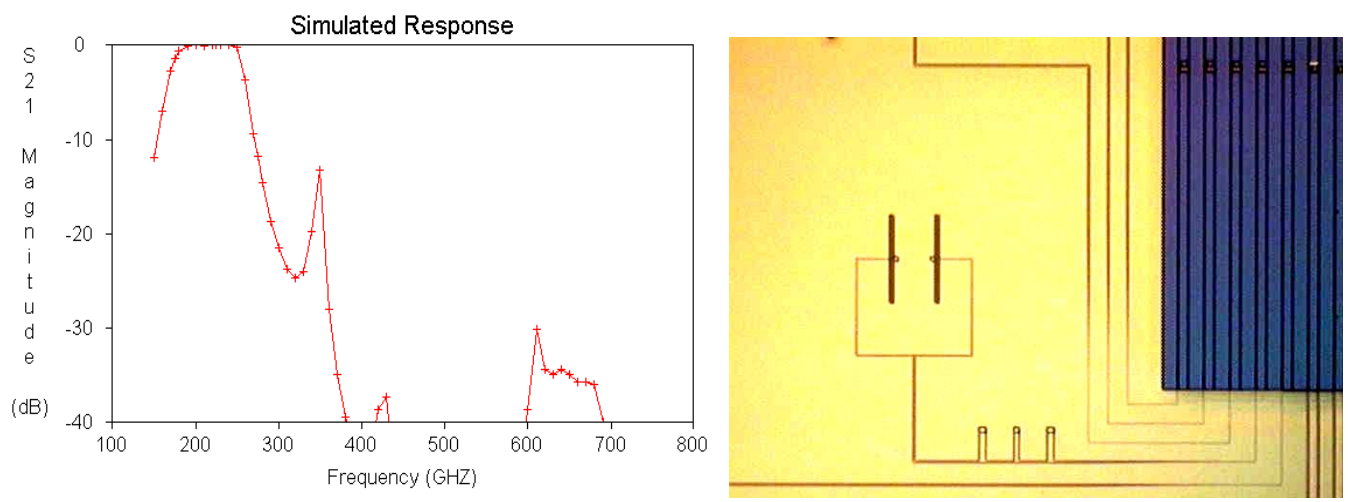

FIGURE 1. Left: Simulated response of combined bandpass \& lowpass filter Right: Photo of a prototype antenna coupled bolometer. The dual slot antenna, bandpass filter, and suspended TES are visible. This particular pixel does not have the lowpass filter.

Our design uses a three-pole bandpass filter centered at $217 \mathrm{GHz}$ in conjunction with a low-pass filter to suppress the spurious pass-bands of the band-pass filter. The initial design work was done with transmission line circuit-simulator software[7] and the final design was checked in a full wave em simulator[8] (Fig 1). The kinetic inductance of the niobium will cause a significant deviation from the expected response of a filter made with a zero resistance normal metal. A correction for this effect can be made with a suitable adjustment of material parameters[9]. The microstrip transmission line is terminated in a load resistor, which is in good thermal contact with a transition-edge sensor on a silicon-nitride bridge. Since the thermal conductance between the load resistor and the TES should be much greater than the conductance along the legs of the bridge, energy dissipated in the load resistor will be effectively coupled into the TES. The nitride suspension is similar in design to previously fabricated devices[10] with an estimated thermal conductance of $2 \times 10^{-11} \mathrm{~W} / \mathrm{K}$. 
We have constructed test devices in the UC Berkeley Microlab incorporating these design elements(Figs1,2). Standard microfabrication techniques were used throughout. We plan to begin testing the spectral response and efficiency of the detectors shortly.

\section{ANTENNA-COUPLED ARRAYS}

One of the powerful advantages of the antenna-coupled architecture is that multi-frequency pixels can be achieved with planar RF multiplexers, rather than the bulky filter systems used with horn-coupled arrays. There are many different types of transmission-line multiplexers, but preliminary simulations show that for 2-3 channels even a simple microstrip " $\mathrm{T}$ " can provide good performance. The double-slot dipole antenna has roughly an octave of bandwidth suitable for 2-3 broad photometric bands.

For double-slot dipole antennas, the packing of the focal-plane has a set of trade-offs that are the same as the well-known trade-offs for horn antennas[11]. For horn antennas, a range of aperture sizes can be used. If the focal plane area is held constant, small horn apertures give the highest sensitivity to diffuse sources, Nyquist or better sampling of the sky, and a high number of pixels to instrument. Large horn apertures maximize sensitivity to point sources, undersample the sky (requiring several pointings to get a full map), and give a low number of pixels to instrument. For a double-slot dipole antenna, the effective aperture can be varied with the diameter of the hemispherical dielectric lens that is typically used in direct contact with the antenna. This lens also suppresses substrate modes.

There are a variety of methods for coupling arrays of antennas to a telescope. For antennas on silicon substrates, silicon lenses are typically used to achieve low reflection at the interface to the substrate. Silicon can be machined with a variety of methods, but more work needs to be done to find a straightforward method for manufacturing large lens arrays. The most straightforward arrangement would be to have a lens for each antenna. Such an array of hemispherical lenses would resemble a fly's eye, and has been described in detail by Buttgenbach[12]. Filipovic et al. have pointed out that a single lens can be used for an array of tens of antennas, which would simplify the manufacturing of the lens array[13]. An alternative to using a dielectric lens is to manufacture the antennas on silicon-nitride membranes and use a parabolic reflector behind each antenna as described by Filipovic et al.[14] If the telescope is matched to the $\mathrm{f} / \# \sim 1$ of the membrane-mounted double-slot dipole a simple flat reflector can be used.

To illustrate the potential of the approach we have described, we present a preliminary focal-plane design for a ground-based CMB polarization experiment incorporating dualpolarization double-slot antennas with one or two frequency bands per pixel (Fig 2). Each pixel has a diffraction-limited beamsize, with the highest frequency bands having the smallest beamsizes. Since aberrations are typically smallest at the center of the focal plane, the highest frequency pixels are located near the center. The lowest frequency pixels are positioned towards the outer edge. The $150 \mathrm{GHz}$ channels will have the highest sensitivity and this configuration fills a high fraction of the usable $150 \mathrm{GHz}$ focal plane with $150 \mathrm{GHz}$ pixels.

\section{ACKNOWLEDGEMENTS}

This work was supported in part by the Director, Office of Science, Office of Basic Energy Sciences of the U. S. Department of Energy under Contract No. DE-AC03-76SF00098, by NSF Grant FD97-31200, and by NASA/Ames Grant FDNAG2-1398. All devices were fabricated at the UC Berkeley Microfabrication Laboratory. 

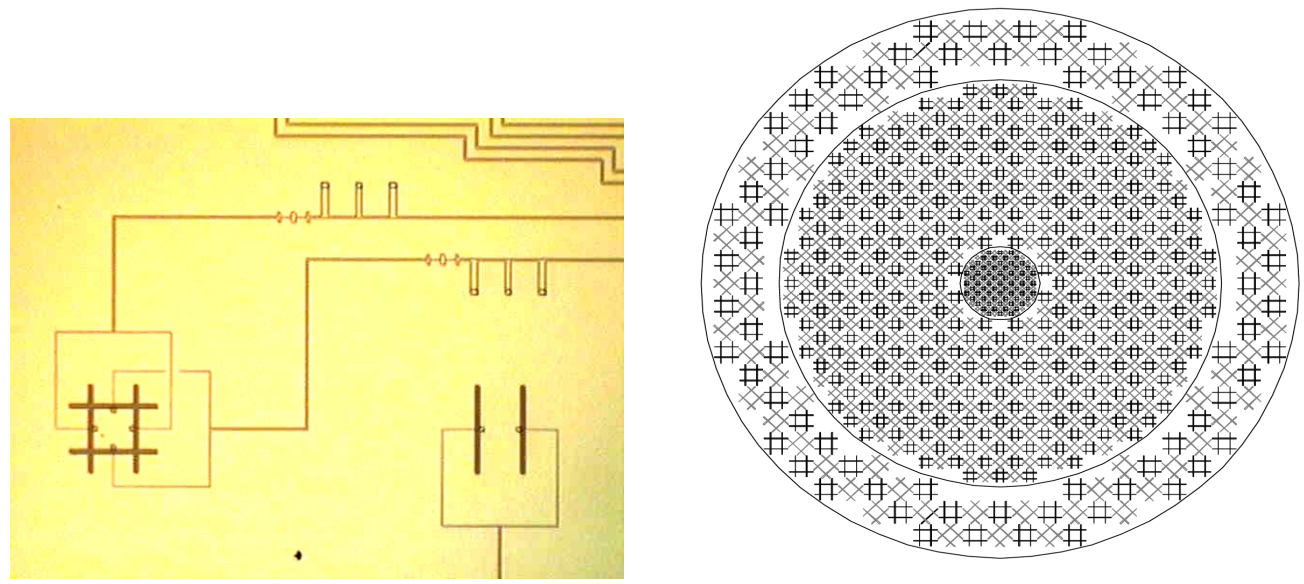

FIGURE 2. Left: Photo of dual-polarization double-slot antenna on test chip with bandpass \& lowpass filters Right: Preliminary design of a focal plane array of bolometers for a ground based CMB polarization experiment. Each pixel is a dual-polarization double-slot dipole antenna. There are 150 pixels in the outside circle with one band at $90 \mathrm{GHz}$. There are 600 pixels in the middle circle with two bands at 150 and $240 \mathrm{GHz}$. There are 150 pixels in the inner circle with bands at 270 and $350 \mathrm{GHz}$

\section{REFERENCES}

1. J. E. Carlstrom et al., "Imaging the Sunyaev-Zel'dovich Effect", Physica Scripta, vol.T85, 2000

2. A. W. Blain et al., "Millimetre/Submillimetre-wave Emission-line Searches for High-redshift Galaxies", Monthly Notices of the Royal Astronomical Society, vol. 313, 2000

3. D. Filipovic, S. Gearhart, and G. Rebeiz, "Double Slot Antennas on Extended Hemispherical and Elliptical Silicon Dielectric Lenses", IEEE Trans. on Microwave Theory and Techniques, vol. 41, no. 10 , October 1993

4. G. Chattopadhyay and J. Zmuidzinas, "A Dual-Polarized Slot Antenna for Millimeter Waves”, IEEE Trans. on Antennas and Propogation, vol. 46, no. 5, May 1998

5. R. L. Kautz, "Picosecond Pulses on Superconducting Striplines", J. of Appl. Phys., 49(1), Jan. 1978

6. D. McGinnis and J. Beyer, "A Broad-Band Microwave Superconducting Thin-Film Transformer", IEEE Trans. on Microwave Theory and Techniques, vol. 36, no. 11, November 1988

7. MMICAD, Optotek LTD. http://www.optotek.com

8. Sonnet em Suite, Sonnet Software, Inc. http://www.sonnetusa.com

9. A.R. Kerr, "Surface Impedance of Superconductors and Normal Conductors in EM Simulators", ALMA Memo No. 245, http://www.alma.nrao.edu/memos/html-memos/alma245.1.pdf

10.J.M. Gildemeister, Adrian T. Lee, P.L. Richards, accepted for publication by Applied Optics

11.J. J. Bock, J. Glenn et al., "Silicon Nitride Micromesh Bolometer Arrays for SPIRE", SPIE Conf. on Advanced Tech. MMW, Radio, and Thz Telescopes, SPIE Proceedings Vol. 3357, 1998

12. T. Buttgenbach, "An Improved Solution for Integrated Array Optics in Quasi-Optical mm and Submm Receivers: the Hybrid Antenna", IEEE Trans. on Microwave Theory and Techniques, vol. 41, no. 10, October 1993

13.D. Filipovic, G. Gauthier, S Raman, and G Rebeiz, "Off-Axis Properties of Silicon and Quartz Dielectric Lens Antennas", IEEE Trans. on Antennas and Propogation, vol. 45, no. 5, May 1997

14.D. Filipovic, W. Ali-Ahmad, and G. Rebeiz, "Millimeter-Wave Double-Dipole Antennas for HighGain Integrated Reflector Illumination", IEEE Trans. on Microwave Theory and Techniques, vol. 40, no. 5, May 1992 